\title{
On the Biphoton Wavelength
}

\author{
P. H. Souto Ribeiro \\ Instituto de Física, \\ Universidade Federal do Rio de Janeiro, \\ Caixa Postal 68528, Rio de Janeiro, RJ, 21945-970, Brazil
}

Received on 30 January, 2001

\begin{abstract}
We report on an experiment showing that the wavelength of a biphoton is clearly dependent on the measurement scheme and on the way it is defined. It is shown that it can take any value, depending on the control of the interferometer phase differences. It is possible to identify the interference of the single and two-photon wavepackets as particular cases of the most general interference process. The variable wavelength has no implication on the energy of the individual photons neither on the total energy of the biphoton.
\end{abstract}

\section{Introduction}

The use of interferometry for measuring the wavelength of a radiation field is probably one of its older applications. Nowadays the concept of interferometry has been extended. It has become possible to observe experimentally the interference for one particle and even for multiparticle wave fields. The two-photon field produced in the parametric down-conversion, have been extensively utilized in many of the so-called multiparticle interferometry experiments[1,2].

In this new type of interference, it is not possible to ignore the quantum aspects of the electromagnetic field. In quantum interferometry, it is also possible to associate interference patterns to the wavelength of a field. For single photon fields, classical and quantum interpretations of the interference experiments lead to the same wavelength. For multi-photon or multi-particle fields however, the wavelength can be dependent on the way the measurement is performed, and a classical interpretation is no longer possible. Thinking of twophoton wavepackets, for example, if we can make the two photons travel together through an interferometer as they were contained in one packet, we can measure a wavelength corresponding to an entity with the energy two times larger than the single photon one[3, 4]. This concept is quite general in quantum physics and it can be extended to any particle or field and the DeBroglie wavelength will be associated to the total energy of the system.

In this paper we study the two photon interference from the point of view of the measurement of the wavelength. We present an experiment whose configuration is capable to produce quantum interference without the use of material interferometers, in the sense that no double-slits and no beam-splitters are used.
It consists of a transverse version of interferometers of the type of Mandel's[5] and Zeilinger's[6]. It is also similar to the interferometer presented by Klyshko et al. in Ref.[7], but without the double-slits and with the possibility of detecting signal and idler photons in completely independence, as it will be shown. This is the main difference from previous transverse interferometers $[8,9,10,11]$. Another experiment recently performed by Fonseca et al.[12], utilizes the same principle for measuring a non-local wavelength for a two-photon wavepacket. The configuration presented here is similar to that presented by White et al.[13] for producing polarization entangled states with high intensities, however in our case the polarization state is not entangled. Twin photons from the parametric down-conversion process are used. These photons have been called biphotons as a reference to their strong correlation at the quantum level. The interference fringes are obtained by measuring coincidence counts and the frequency of the oscillation of the patterns are associated to wavelengths for the biphotons. It is shown that this frequency can be arbitrarily varied, depending on the way the measurements are performed. It is also shown, that the measured wavelengths can be assigned to single and to two-photon wavepackets, for two kinds of measurement. Attempts to connecting the oscillation frequency and the biphoton wavelengths for other kinds of measurements indicate that special care must be taken in defining and measuring wavelengths in multiparticle interferometry. On the other hand, this interferometer can be used in the production and manipulation of position entangled states and has potential for applications in measurements of diffraction indexes. A single mode quantum theory is enough to explain the behavior of the frequency of the patterns and it is in agreement with the experimental data. 


\section{The two-photon interference}

Let us analyze the situation sketched in Fig. 1. The pump laser passes through two nonlinear crystals, labeled crystal 1 and crystal 2. Twin photons can be produced in each one of the crystals. Signal and idler photons produced in crystal 1 are directed to detectors A and B respectively, so that coincidence between signal and idler channels can be measured. Suppose that degenerate photon pairs produced in crystal 2, can also be directed to the same detectors. This condition is simply fulfilled by tilting crystal 2 relatively to the vertical axis.

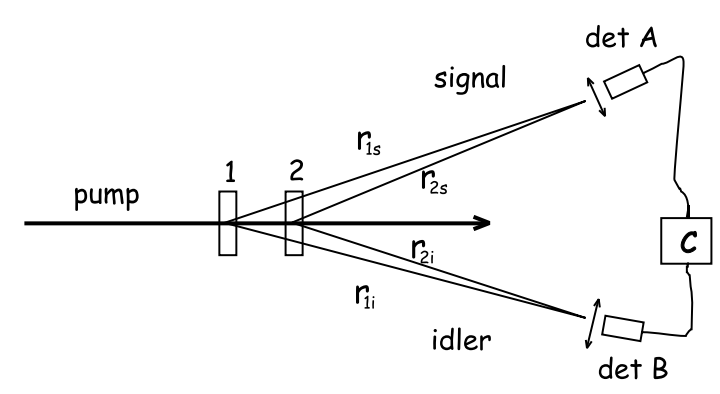

Figure 1. Outline of the experiment.

The situation described above is suitable for quantum interference. Note that the time of emission of photon pairs cannot be specified, since it is a spontaneous emission and the coherence length of the pump laser is larger than the distance between crystals. In this case, coincidence counts produced by photon pairs originated in crystal 1 are indistinguishable from those of crystal 2. Interference fringes in the coincidence counting rate can be observed, as long as the phase difference between these two probabilities is varied. As each crystal works like an extended source, no interference is observed for the individual intensities.

This interference process can be described in a simplified form with the use of a monomode quantum approach. It is enough to explain the main properties of the coincidence patterns, including the effective wavelength. However, for taking into account for the degree of coherence and its consequences in the visibility of the fringes, a multi-mode theory would be necessary. In this work, we will restrict ourselves to the simpler case.

The quantum state of the field produced by both crystals is given by:

$$
|\Psi\rangle=\frac{1}{\sqrt{2}}\left(|1\rangle_{i 1}|1\rangle_{s 1}|0\rangle_{i 2}|0\rangle_{s 2}+|0\rangle_{i 1}|0\rangle_{s 1}|1\rangle_{i 2}|1\rangle_{s 2}\right) .
$$

The electric field operators for signal and idler modes at the detection planes are given by:

$$
\begin{gathered}
E_{A}^{(+)}=\mathrm{a}_{1 s} \mathrm{e}^{-i\left(\phi_{1 s}+\mathrm{kr}_{1 s}\right)}+\mathrm{a}_{2 s} \mathrm{e}^{-i\left(\phi_{2 s}+\mathrm{kr}_{2 s}\right)} ; \\
E_{B}^{(+)}=\mathrm{a}_{1 i} \mathrm{e}^{-i\left(\phi_{1 i}+\mathrm{kr}_{1 i}\right)}+\mathrm{a}_{2 i} \mathrm{e}^{-i\left(\phi_{2 i}+\mathrm{kr}_{2 i}\right)},
\end{gathered}
$$

where $\phi_{j x}$ is the phase of the field at the emission point, with $\mathrm{j}=1,2$ and $x=\mathrm{s}, \mathrm{i}$ and $\mathrm{k}$ is the wave number for both signal and idler modes. $\mathrm{r}_{j x}$ is the distance between crystal $j$ and the detector at the $x$ side.

The coincidence counting rate can be easily calculated:

$$
\begin{aligned}
C & \left.=\left|E_{A}^{(+)} E_{B}^{(+)}\right| \Psi\right\rangle\left.\right|^{2} \\
& =2\left[1+\cos \left(\phi_{1 i}+\phi_{1 s}+\mathrm{kr}_{1 i}+\mathrm{kr}_{1 s}\right.\right. \\
& \left.\left.-\phi_{2 i}-\phi_{2 s}-\mathrm{kr}_{2 i}-\mathrm{kr}_{2 s}\right)\right]
\end{aligned}
$$

From the phase matching conditions we have that $\phi_{1 i}+\phi_{1 s}=\phi_{1 p}$ and $\phi_{2 i}+\phi_{2 s}=\phi_{2 p}$, where $\phi_{1 p}$ and $\phi_{2 p}$ are the pump laser phases at crystals 1 and 2 respectively. We see that one condition for observing interference is that $\phi_{1 p}-\phi_{2 p}=$ const. That is to say the coherence length of the pump laser must be larger than the distance between crystals.

Eq. 3 can be put in the form:

$$
C=2\left\{1+\cos \left[\mathrm{k}\left(\delta_{i}+\delta_{s}\right)+\phi\right]\right\}
$$

where

$$
\begin{aligned}
\phi & =\phi_{1 p}-\phi_{2 p}+\mathrm{k}\left(\overline{\mathrm{r}}_{1 i}+\overline{\mathrm{r}}_{1 s}-\overline{\mathrm{r}}_{2 i}-\overline{\mathrm{r}}_{2 s}\right) ; \\
\mathrm{r}_{1 i} & =\overline{\mathrm{r}}_{1 i}+\delta_{1 i} ; \\
\mathrm{r}_{1 s} & =\overline{\mathrm{r}}_{1 s}+\delta_{1 s} ; \\
\mathrm{r}_{2 i} & =\overline{\mathrm{r}}_{2 i}+\delta_{2 i} ; \\
\mathrm{r}_{2 s} & =\overline{\mathrm{r}}_{2 s}+\delta_{2 s} ; \\
\delta_{i} & =\delta_{1 i}-\delta_{2 i} ; \\
\delta_{s} & =\delta_{1 s}-\delta_{2 s} .
\end{aligned}
$$

With the aid of Eqs. 3 and 4 it is clearly seen that the interference fringes are sensible to phases that depend on the paths from crystals 1 and 2 to detectors. It is worth noting that phase $\delta_{i}$ can be varied independently from $\delta_{s}$. Displacing signal or idler detector one can vary each one of these phases. Consider the case where $\delta_{i}=\alpha \delta_{s}$. In this case, the variable phase in Eq. 4 can be written as:

$$
C=2\left\{1+\cos \left[\mathrm{k}(1+\alpha) \delta_{s}+\phi\right]\right\} \text {. }
$$

The parameter $\alpha$ in the above equation can assume any value and we demonstrate experimentally in this paper that it can be easily controlled. In fact, $\alpha$ is the ratio between displacements of detectors $\mathrm{A}$ and $\mathrm{B}$. 


\section{The Experimental Set-up}

The experimental set-up shown in Fig. 1 was implemented with a c.w. He-Cd laser operating at $442 \mathrm{~nm}$. The output power was about $200 \mathrm{~mW}$. It was used to pump two $1 \mathrm{~cm}$ long $\mathrm{LiIO}_{3}$ crystals. The distance between crystals was around $2 \mathrm{~cm}$ and the distance between crystal 1 and both detectors A and B was nearly $1.5 \mathrm{~m}$. Crystals were cut for collinear degenerate downconversion, that is to say the optical axis at 37.3 degrees relatively to the input/output faces. In order to make degenerate beams emerge from the crystal at angles different from zero, it was necessary to tilt it slightly relatively to the vertical direction. In our case, the direction of propagation of the twin beams were nearly 7 degrees with the pump beam direction. The output angle of the beams produced in crystal 2 were slightly bigger, in order to achieve superposition at the detectors with the beams originated at crystal 1 .

The detectors were avalanche photodiodes inside photon counting modules (SPCM-AQ / EG\&G). The incoming light passes through a small vertical slit (about $0.5 \mathrm{~mm}$ ) and an AR coated lens with $25.4 \mathrm{~mm}$ focal length, before reaching the active detection area of about $0.2 \mathrm{~mm}$. The modules are mounted on $\mathrm{X}$ $\mathrm{Y}$ translation stages, so that the transverse detection plane can be scanned with up to $5 \mu \mathrm{m}$ resolution. The output pulses were sent to counters (SR-400 and SR-620 / SRS), where single rates were counted and the coincidence logic was implemented. Counters were controlled by a microcomputer which was used to save data.

\section{Experimental Results}

We have carried out coincidence interference patterns for several values of the parameter $\alpha$ in Eq. 6. For doing so, we have simply changed the relative displacement between detectors $\mathrm{A}$ and $\mathrm{B}$.

For $\alpha=0$, detector B was kept fixed, while detector A was scanned transversally in the horizontal plane. The single photon and the coincidence counting rates were then registered. The results are shown in Fig. 2. While the singles show a nearly gaussian profile, the coincidences show interference fringes. The visibility and the wavevector of the coincidence fringes were obtained by a nonlinear curve fitting with the usual function for the double-slit interference. The wavevector for $\alpha=0$ will be called $\mathrm{k}_{0}$. It is associated to the single photon interference. Note that the absolute value of $\mathrm{k}_{0}$ depends on the geometry. In the analogy with a double-slit experiment, each crystal correspond to one slit. However, the light is not emitted in the direction that would correspond to a central(zero order) maximum. It is emitted in a direction corresponding to a higher order. This means that the associated wavelength is multiplied by a large integer, which is not important in this work. For this reason the wavevectors will be presented in arbi- trary units. The procedure is repeated keeping detector A fixed and scanning detector B. The result shows a profile similar to that of Fig. 2, showing the symmetry between scans with one of the detectors fixed. These patterns can be interpreted as single photon wavepacket interference ones. This is a consequence of the fact that only signal or idler paths are changed, when only signal or idler detectors are moved individually.

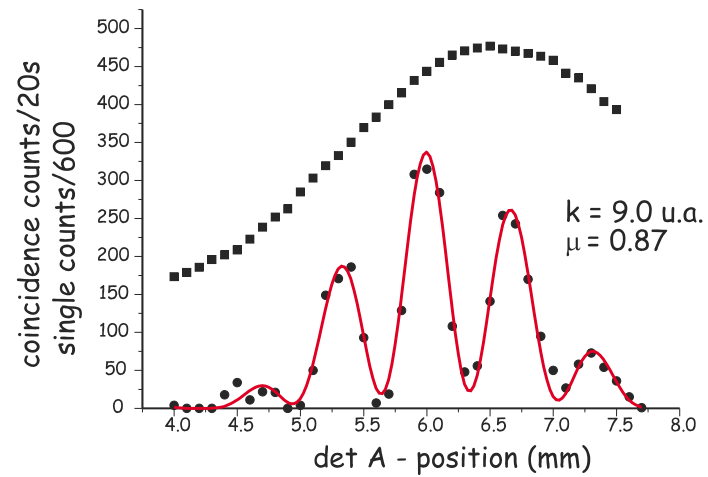

Figure 2. Singles and coincidence profile for $\alpha=0$. Detector $\mathrm{A}$ is scanned and detector B is fixed.

For $\alpha=+1$, detectors $\mathrm{A}$ and $\mathrm{B}$ were simultaneously displaced with the same velocity. This is equivalent to saying that the detectors were displaced with equal steps. The result is shown in Fig. 3. The convention used establishes that the positive $\alpha$ implies in additive phase shifts in signal and idler sides. In the experiment, this condition was achieved by displacing both detectors towards the pump beam. The visibility and the wave number, that gives the frequency of the oscillations, were set as free parameters in the fittings. As a result, we observe that the wave number for the curve in Fig. 3 is two times the one in Fig. 2. $\mathrm{k}_{+1}($ signal side $)=\mathrm{k}_{+1}($ idler side $)=2 \mathrm{k}_{0}$. This was predicted by Eq. 6 for $\alpha=+1$ and it works also for $\alpha=-3$. The pattern of Fig. 3 can be interpreted as a two-photon wavepacket interference. We will address this point again in next section.

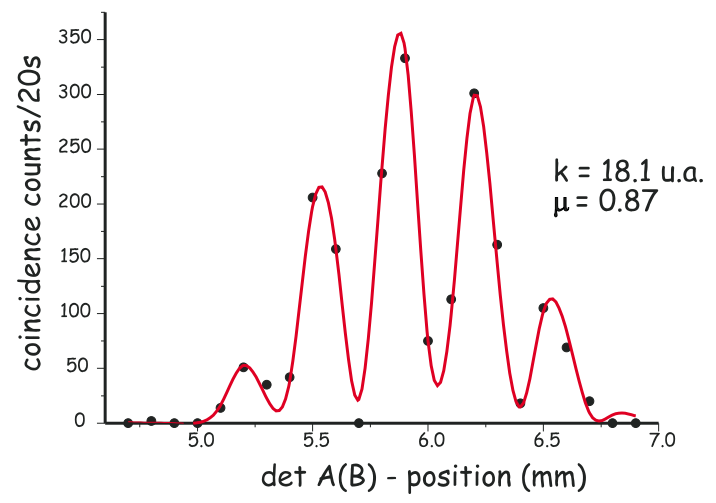

Figure 3. Coincidence profile for $\alpha=+1$. Detector A and $\mathrm{B}$ are scanned simultaneously with the same velocity.

For $\alpha=+\frac{1}{2}$, detector $\mathrm{A}$ was displaced two times slower than detector $\mathrm{B}$. The displacement is performed 
so that detectors get together to the position corresponding to the coincidence peak detection in previous measurements. With this procedure it is possible to take care for the symmetry of the interference curve. The results are shown in Fig. 4. In Fig. 4a the coincidence counts are plotted as a function of the detector A position and in Fig. 4b, the same coincidence counts are plotted as a function of the detector B position. This is necessary now, because the displacements are different, we do not have a common coordinate anymore, as in previous cases. The fitting of the curves lead to $\mathrm{k}_{+\frac{1}{2}}$ (signal side) $=\frac{3}{2} \mathrm{k}_{0}$, which corresponds to $\alpha=+\frac{1}{2}$ and $\mathrm{k}_{+\frac{1}{2}}$ (idler side) $=3 \mathrm{k}_{0}$ which corresponds to $\alpha_{i}=+2$ when the phase shift is written in terms of the idler coordinates. This is in agreement with Eq. 6 .
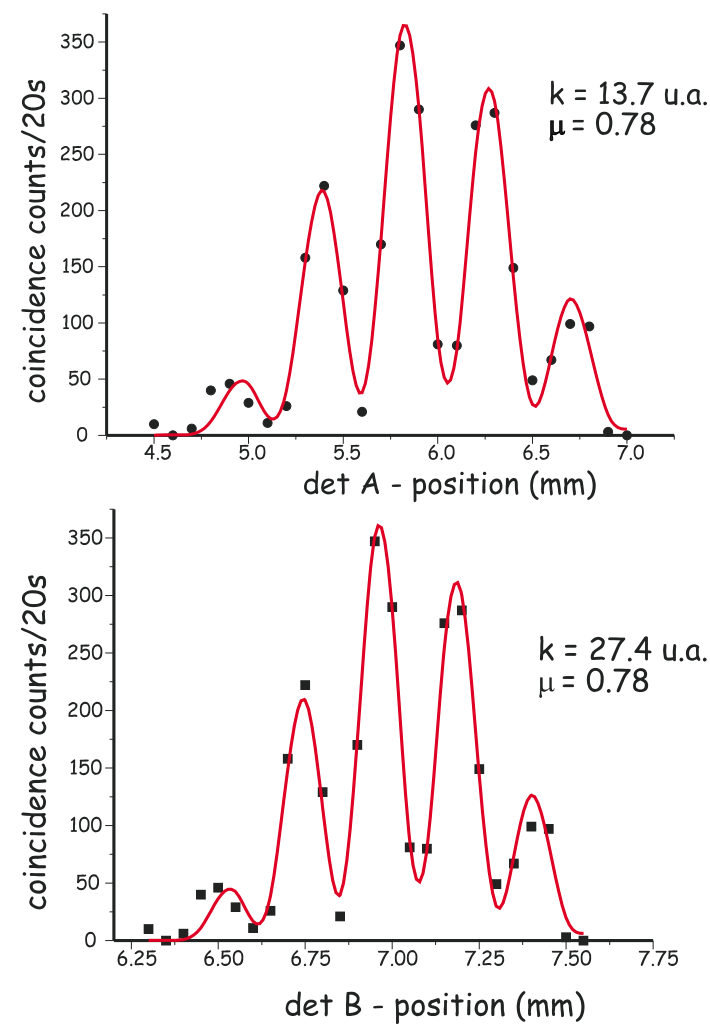

Figure 4. Coincidence profiles for $\alpha=+\frac{1}{2}$. Detector A and $\mathrm{B}$ are scanned simultaneously with different velocities.

For $\alpha=-\frac{1}{2}$, detector $\mathrm{A}$ is still displaced two times slower than detector $\mathrm{B}$, but now the negative sign indicates that the sense of one of the displacements is changed. Detector A moves towards the pump beam while detector B move backwards the pump beam. The results are shown in Fig. 5. In Fig. 5a the coincidence counts are plotted as a function of the detector A position while in Fig. 5b they are plotted as a function of the detector B position. From the point of view of detector $\mathrm{A} \alpha=-\frac{1}{2}$ and the wavevector is $\mathrm{k}_{-\frac{1}{2}}$ (idler side) $=\frac{1}{2} k_{0}$. From the point of view of detector B $\alpha_{s}=-2$ and $\mathrm{k}_{-\frac{1}{2}}$ (signal side $)=k_{0}$.
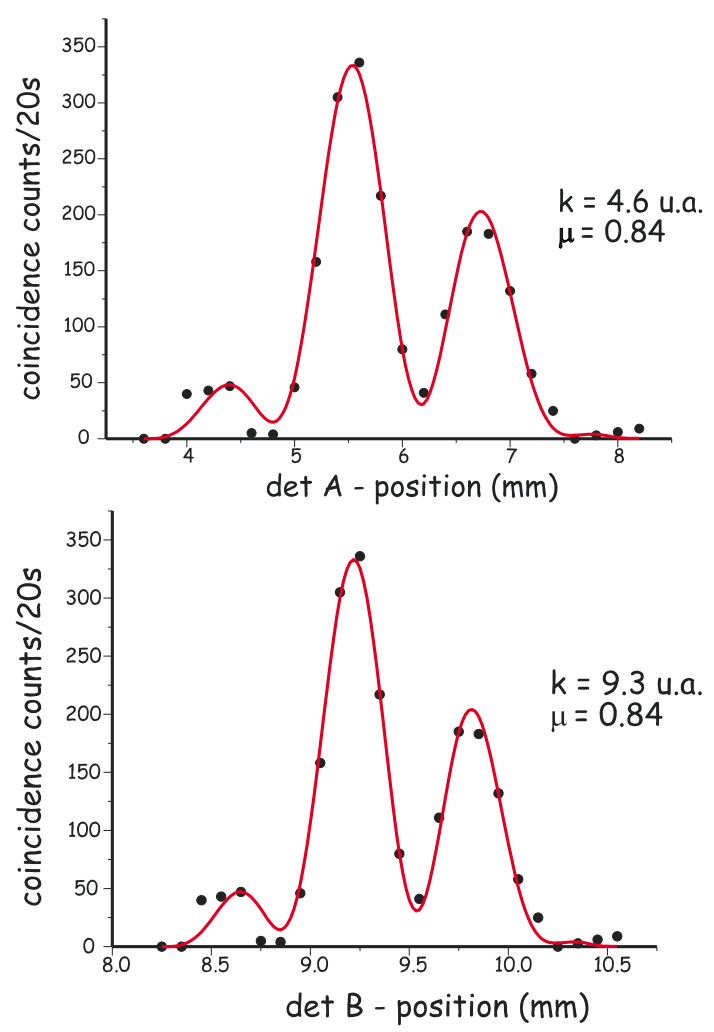

Figure 5. Coincidence profiles for $\alpha=+\frac{1}{2}$. Detector A and $\mathrm{B}$ are scanned simultaneously with different velocities.

\section{Discussion}

From Eq. 6 and experimental results presented in previous section, it is clear that the wavelength associated to the coincidence patterns can be continuously varied and that it can assume any value, even a fraction or a multiple of the single photon one. This fact can be explained by the phase entanglement between signal and idler photons. It also seems that it has no implications on the energy of the individual photons neither on the total energy of the biphotons. It is clear from all experiments utilizing twin photons from the parametric downconversion, that the process behind all quantum effects is entanglement. The entanglement is a consequence of a process that we could call transfer of spectrum from the pump beam to the biphotons. A particular case of that is the transfer of angular spectrum described in Ref. [11] dealing with the transverse degrees of freedom of the field. In the experiment we present here, it is nice to be able to understand the main features in connection with entanglement and transfer of angular spectrum for a simple case utilizing a monomode approach. This is one of the virtues of the interferometer presented.

It is interesting however, to analyze some possible interpretations. When $\alpha=0$, one detector is fixed and the other one is moved. In this case, the experiment 
can be viewed as a single photon wavepacket interference because the phase difference depends only on trajectories for the same photon. When $\alpha=+1$ or $\alpha=-2$, both detectors are moved simultaneously with the same velocity. In this case, the experiment corresponds to an unfolded version of a two-photon wavepacket interference. Note that in one of the previous experiments[4], it was necessary to prepare the state of the field by manipulating the pump beam, in order to avoid single photon interference. In the present configuration, each crystal plays the role of one slit in the analogy with a double slit experiment. However, each twin photon pair is always (the probability is much bigger) emitted by the same crystal and never (the probability is much smaller) by different ones. This corresponds to having both photons passing through one of the slits and never one photon through each slit. For this reason, it is not necessary to change the pump laser beam profile. But the analogy is complete.

When $\alpha=+\frac{1}{2}$, for example, it is not possible to assign some physical meaning to the wavelength observed by one of the detectors anymore. For the same set of measurements, the wavevector is three times larger than the single photon one (corresponding to a wavelength three times smaller than the single photon one) from the point of view of the conjugated detector. This result shows that assigning a wavelength to something we call biphoton can be dangerous. The energy of each individual photon is not changed during the interference process, neither during the detection process, and the discussion about this apparent wavelength may turn into speculation. However, diffraction properties are actually changed and that may have consequences in imaging and other applications. Some possible applications can be envisaged. For example, the coincidence patterns could be used for measuring the refraction index for some material placed in the path of the beams. Differential measurements could be performed in a collinear configuration with both beams passing through the sample. We could also think of twin beams with crossed polarizations used for birefringence measurements. The interferometer is quite useful for fundamental research on the production, detection and manipulation of entangled states. Recently, it was used in our laboratory for producing position entangled states wich were coupled to polarization entanglement so that we were able to measure the plarization entanglement thorugh position interference[14]. Experiments currently in progress show that it is also suitable for studying quantum distilation, purification and decoherence.

\section{Conclusion}

A two-photon interferometer without double-slits and without beam-splitters is presented. The frequency of the oscillations of the coincidence interference patterns is varied. These frequencies are associated to the single and to the two-photon wavepackets. Frequencies that are fractions and multiples of the single photon one are observed, in agreement with theory. These variable frequency oscillations are well understood in terms of the quantum theory and two particle entanglement. Some applications in the new field of quantum imaging and measurements of refraction indexes are promising possibilities. The interferomenter is being used in experiments for producing and manipulating entangled states. The entangled states are essential in the field of quantum information.

\section{Acknowledgment}

We thank Drs. Carlos Monken and Sebastião de Pádua for lending one of the detectors and also for many helpfull discussions. Financial support was provided by Brazilian agencies $\mathrm{CNPq}$, PRONEX, FAPERJ and FUJB.

\section{References}

[*] Corresponding author. E-mail address: phsr@if.ufrj.br

[1] Greenberger, Horne and Zeilinger, Phys. Today 8, 22 (1993)

[2] Anton Zeilinger, Review of Modern Physics 71, S288 (1999)

[3] J. Jacobson, G. Björk, I. Chuang and Y. Yamamoto, Phys. Rev. Lett. 74, 4835 (1995)

[4] E. J. S. Fonseca, C. H. Monken and S. Pádua, Phys. Rev. Lett. 82, 2868 (1999)

[5] X. Y. Zou, L. J. Wang and L. Mandel, Phys. Rev. Lett. 67, $318(1991)$

[6] T. J. Herzog, J. G. Rarity, H. Weinfurter and A. Zeilinger, Phys. Rev. Lett. 72, 629 (1994)

[7] A. V. Burlakov, D. N. Klyshko, S. P. Kulik and M. V. Chekova, JETP Lett. 69, 831 (1999)

[8] P. H. Souto Ribeiro, S. Pádua, J. C. Machado da Silva, and G. A. Barbosa, Phys. Rev. A 49, 4176 (1994).

[9] P. H. Souto Ribeiro and G. A. Barbosa, Phys. Rev. A 54, 3489(1996).

[10] E. J. da Silva Fonseca, P. H. Souto Ribeiro, S. Pádua and C. H. Monken, Phys. Rev. A 60, 1530 (1999).

[11] C. H. Monken, P. H. Souto Ribeiro and S. Pádua, Phys. Rev. A 573123 (1998).

[12] E. J. S. Fonseca, Z. Paulini, P. Nussenzveig, C. H. Monken, and S. Pádua, Phys. Rev. A 63, 043819 (2001)

[13] A. G. White, D. F. V. James, P. H. Eberhard and P. G. Kwiat, Phys. Rev. Lett. 83 , 3103 (1999)

[14] M. França Santos, P. Milman, A. Z. Khoury, and P. H. Souto Ribeiro, Phys. Rev. A 64, 023804 (2001). 